\title{
Foliculitis perforante: una dermatosis de eliminación transepidérmica
}

\section{Sebastiân Cardemil1 ${ }^{1}$, Carolina Pardo ${ }^{2}$, Héctor Fuenzalida ${ }^{3}$, Laura Segovia ${ }^{4}$}

1. Médico dermatólogo y venereólogo, Hospital El Pino, Santiago de Chile, Chile

2. Residente de Dermatología, Unisanitas- Hospital Centro Dermatológico Federico Lleras Acosta. Bogotá, Colombia

3. Médico dermatólogo y venereólogo, Hospital El Pino, Santiago de Chile, Chile

4. Médico anatomo-patólogo, Hospital Barros LucoTrudeau, Santiago de Chile, Chile

\section{RESUMEN}

Las dermatosis perforantes son un grupo de enfermedades pápulo-nodulares poco frecuentes, caracterizadas por formación de tapones queratósicos en los cuales los componentes de la matriz dérmica migran hacia la epidermis para ser eliminados sin un factor desencadenante. Se clasifican en primarias y secundarias. Se asocian a enfermedades sistémicas crónicas, y alteraciones de las fibras de colágeno y las elásticas, entre otros.

Se presenta un caso de foliculitis perforante, como una dermatosis perforante primaria, para su diagnóstico y tratamiento.

PALABRAS CLAVE: dermatosis; epidermis; foliculitis; folículo piloso.

\section{SUMMARY}

Perforating dermatoses are a group of papular nodular rare diseases characterized by the formation of keratotic plugs in which dermal matrix components migrate into the epidermis to be removed without a trigger. They are classified into primary and secondary. They are associated with chronic systemic diseases, changes in collagen and elastic fibers, among others.

Here is a case of perforating folliculitis, as a primary perforating dermatosis, for diagnosis and treatment.

KEY WORDS: Dermatoses, epidermis, folliculitis, hair follicle.

\section{INTRODUCCIÓN}

Dado el difícil manejo de las dermatosis perforantes, es importante poder reconocerlas, diagnosticarlas y diferenciarlas. Los trastornos perforantes primarios de la piel se caracterizan por la eliminación transepidérmica de algunos componentes de la dermis y se clasifican en cuatro tipos: elastosis perforante serpiginosa, colagenosis perforante reactiva, foliculitis perforante y enfermedad de Kyrle ${ }^{(1)}$.El tejido elimi-
Correspondencia:

Sebastián Cardemil

Carolina Pardo

Email:

sebastian.cardemilb@gmail.com carolina.pardo@hotmail.com

Recibido: 11/05/2016

Aceptado: 18/11/2016

Conflictos de interés:

No se reportan conflictos de interés. 


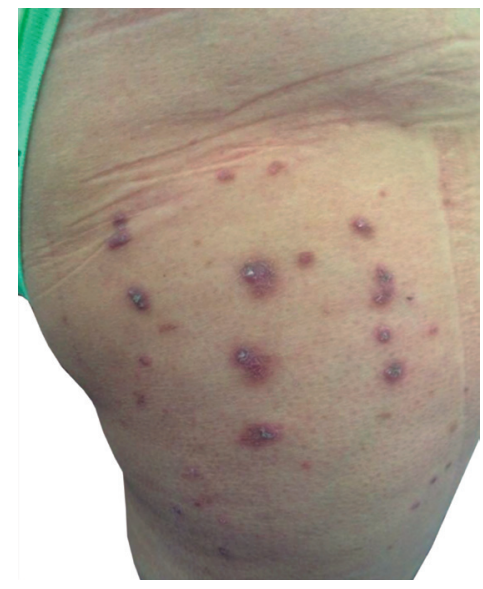

FIGURA 1. Foliculitis perforante. Pápulas de color pardo-violáceas, con hiperqueratosis central y liquenificación.

nado, sin una causa específica, puede ser fibras elásticas, colágeno, queratina, detritos celulares o células inflamatorias. La foliculitis perforante se caracteriza por la expulsión de material dérmico alterado a través de los infundíbulos foliculares ${ }^{(2)}$.

\section{CASO CLÍNICO}

Se trata de una paciente de sexo femenino de 47 años de edad, con un cuadro clínico de dos años de evolución de lesiones pruriginosas en el glúteo y el muslo derechos. Como antecedente, refirió diabetes mellitus no controlada (hemoglobina 'glucosilada' de 9,6\%) en tratamiento con 18 UI de insulina lenta, hipotiroidismo con suplemento hormonal de $50 \mu \mathrm{g}$ diarios de levotiroxina y amputación traumática del pie derecho.

En el examen físico, se observaron múltiples pápulas de color pardo-violáceas, con hiperqueratosis central y liquenificación, en el glúteo y la cara posterior del muslo del miembro inferior derecho (figura 1). Inicialmente, se planteó el diagnóstico de liquen ruber plano y se practicó una biopsia en la que se reportó foliculitis perforante (figura 2). Se inició tratamiento con $10 \mathrm{mg}$ diarios de isotretinoína oral, y se obtuvo una respuesta terapéutica parcial después de tres meses.

\section{DISCUSIÓN}

La foliculitis perforante se caracteriza por la alteración de la porción infundibular de la pared folicular, con eliminación transepidérmica de detritos celulares y tejido conjuntivo. Fue descrita por Mehregan y Coskey en 1968, como una erupción folicular queratósica discreta, que compromete las extremidades, caracterizada por disrupción y perforación del infundíbulo folicular (3).
Se caracteriza clínicamente por pápulas foliculares, queratósicas, generalmente pruriginosas, en áreas cubiertas de pelos, de preferencia en las extremidades ${ }^{(4)}$. Puede aparecer a cualquier edad, con una media entre la segunda y la cuarta décadas de la vida, sin predominio según sexo (1). Este cuadro clínico era compatible con los hallazgos encontrados en el examen físico de la paciente, aunque se encontraba fuera del grupo etario descrito en la literatura.

La histopatología se caracteriza por un folículo piloso dilatado con presencia de material ortoqueratósico y paraqueratósico entremezclado con restos basofílicos, compuestos por colágeno necrótico, células inflamatorias y fibras elásticas alteradas muy eosinofílicas. Existen una o varias áreas de perforación folicular en la zona infundibular y, también, es posible encontrar pelos incurvados ${ }^{(5)}$.En el presente caso, se evidenció dilatación del infundíbulo por la presencia de queratina y detritos celulares en la tinción de hematoxilina y eosina y en la de Masson, junto con la migración de las fibras de colágeno hacia la cavidad del infundíbulo (figura 2, A y B).

Se ha sugerido que el trauma es el mecanismo implicado más importante, ya que las lesiones ocurren en las zonas de extensión y de fricción crónica, lo cual aumenta la queratinización (4-6); esta distribución se observó en el presente caso, en el cual las lesiones estaban ubicadas en el glúteo y la región posterior del muslo derecho, expuestos a roce y fricción constante.

La foliculitis perforante se asocia con enfermedades concomitantes como insuficiencia renal crónica, diabetes mellitus, hipertensión arterial sistémica, hepatitis, aterosclerosis, acantosis nigricans, psoriasis, HIV y colangitis esclerosante ${ }^{(1,6,7,8)}$.Dicha asociación se evidencia 
por el antecedente de diabetes mellitus en este paciente, no así con la enfermedad renal, puesto que la creatinina era normal (o,98 mg/dl). Por otra parte, se ha visto que en pacientes que reciben inhibidores del factor de necrosis tumoral alfa (TNF- $\alpha$ ), se estimulan el factor de crecimiento beta (TGF- $\beta$ ) y la hiperqueratinización ${ }^{(9)}$. También, se ha relacionado con el formaldehído presente en tinturas de ropa ${ }^{(6)}$.

Entre los diagnósticos diferenciales se encuentran las dermatosis perforantes clásicas, la queratosis pilar, el queratoacantoma eruptivo, la pitiriasis liquenoide y varioliforme, las erupciones acneiformes y la foliculitis por gramnegativos. De igual forma, deben tenerse en cuenta aquellas enfermedades relacionadas con el prurito urémico, como son liquen plano, porfiria, calcifilaxia y prurigo nodular, en este último caso, por la presencia de lesiones papulonodulares pruriginosas ${ }^{(10,11)}$.
Se han usado múltiples tratamientos, pero, en general, ninguno efectivo o estandarizado. Se han utilizado corticoides tópicos, corticoides sistémicos y fotoquimioterapia (PUVA) ${ }^{(12)}$. En una serie del 2004, se usó UVB debanda estrecha (UVB-NB), durante dos a tres semanas, con una dosis inicial de $400 \mathrm{~mJ} / \mathrm{cm} 2 \mathrm{yun}$ aumento máximo de $1.500 \mathrm{~mJ} / \mathrm{cm} 2$, y se obtuvo resolución de las lesiones después de 10 a 15 sesiones; sin embargo, hubo recurrencia después del tratamiento ${ }^{(13)}$. Se ha reportado el uso de ácido retinoico al o,05\% dos veces al día, con mejoría completa después de cinco meses ${ }^{(4)}$. También, se han usado retinoides orales ${ }^{(12)}$, tratamiento que iniciamos en este caso, con respuesta parcial después de tres meses. De igual forma, se ha usado alopurinol en dosis de $100 \mathrm{mg}$ diarios en pacientes diabéticos o con enfermedad renal crónica, con mejoría entre las dos y
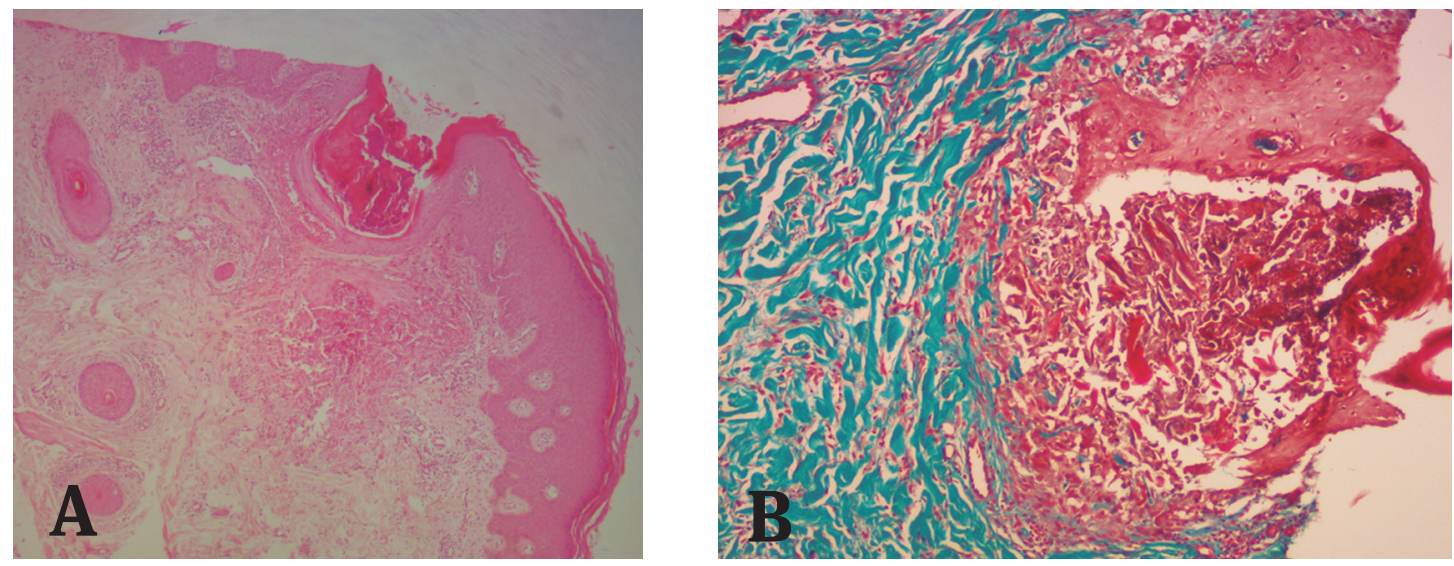

FIGURA2. A infundibulo dilatado con queratina y detritus celulares. (H-E 4X)

B. En verde, se observa la migracion de las fibras colagenas hacia la cavidad del infundibulo (Masson 10X)

cuatro semanas de tratamiento $^{(14)}$. En casos aislados, se ha usado talidomida, $100 \mathrm{mg}$ diarios durante seis meses, con resolución total de las lesiones ${ }^{(4)}$.

\section{CONCLUSIÓN}

Dado el amplio espectro de las dermatosis perforantes, es importante reconocer a la foliculitis perforante como una entidad asociada a enfermedades sistémicas crónicas, especialmente, diabetes mellitus, enfermedad renal crónica y traumatismo repetitivo. Esta dermatosis debe sospecharse al enfrentar lesiones papulares y su diagnóstico debe documentarse adecuadamente con apoyo precoz en el estudio de histopatología.

\section{REFERENCIAS}

1. Santamaría V, Cervantes AM, Barrios E. Dermatosis con eliminación transepidérmica clásicas. Rev Cent Dermatol Pascua. 2002;11:40-8.

2. Pérez OG, Villoldo MS, Schroh RG. Pápulas pruriginosas en zonas pilosas. Foliculitis perforante. Dermatol Argent. 2008;14:400-2.

3. Mehregan AH, Coskey RJ. Perforanting follicutis. Arch Dermatol. 1968;97:394-9.

4. Brodercen MJ, Romano S, Soler S. Foliculitis perforante. Arch Argent Dermatol. 2012;62:110-3.

5. Saray Y, Seçkin D, Bilezikçi B. Acquired perforating derma- 
tosis: Clinico-pathological features in twenty-two cases. J Eur Acad Dermatol Venereol. 2006;20:679-88.

6. Patterson WJ. The perforating disorders. J Am Acad Dermatol. 1984;10:561-81.

7. Patterson JW, Graff GE, Eubanks SW. Perforating folliculitis and psoriasis. J Am Acad Dermatol. 1982;7:369-76.

8. Rubio FA, Herrans P, Robayna G, Peña JM, Contreras F, Casado M. Perforating folliculitis: Report of a case in an HIV infected man. J Am Acad Dermatol.1999;40:300-2.

9. Gilaberte Y, Coscojuela C, Vázquez C, Rosello R, Vera J. Perforating folliculitis associated with tumour necrosis factor alpha inhibitors administered for rheumatoid arthritis. Br J Dermatol. 2007;156: 368-71.

10. Cárdenas C, Bello C, Uribe P. Colagenosis perforante reactiva adquirida. Caso clínico. Rev Med Chile. 2010;138:1281-4.

11. Korula A, Thomas M, Noronha, J. Acquired perforating dermatosis: An innocuous lesion with possibly ominous implications. Cutis. 2010;86:242-4.

12. Zachariae H, Sogaard H. Progressive generalized perforating folliculitis. Dermatologica. 1984;168:131-7.

13. Ohe S, Danno K, Sasaki H, Isei T, Okamoto H, Horio T. Treatment of acquired perforating dermatosis with narrow band ultraviolet B. J Am Acad Dermatol. 2004;50:892-4.

14. Iyoda M, Hayashi F, Kuroki A, Shibata T, Kitazawa K, Sugisaki $\mathrm{T}$, et al. Acquired reactive perforating collagenosis in a nondiabetic hemodialysis patient: Successful treatment with allopurinol. Am J Kidney Dis. 2003;42:E11-3. 